\title{
The Practice of Teledermatology Before, During, and After the COVID-19 Pandemic
}

\author{
Jayarkar Thomas, MD, DD, $\mathrm{PhD}$, DSc \\ Department of Dermatology and Venereology, Chettinad Hospital and Research Institute, Chennai, India
}

\section{Corresponding Author:}

Jayarkar Thomas, MD, DD, PhD, DSc

Department of Dermatology and Venereology

Chettinad Hospital and Research Institute

Rajiv Gandhi Salai

Kelambakkam

Chennai, 603103

India

Phone: 914447411000

Email: jayakarthomas@gmail.com

\begin{abstract}
Background: Since the beginning of the COVID-19 pandemic, the use of telemedicine has quickly expanded in many countries as clinical frameworks have been forced to shift to virtual platforms to guarantee the safety of patients and staff. Teledermatology, specifically, is well-suited for telemedicine, with evidence supporting its viability, even-handed quality and precision, and cost adequacy in comparison to in-person visits. Teledermatology holds extraordinary potential for expanding access to patients and guaranteeing coherence of care, especially for those from rural and underserved regions.

Objective: The aim of this research is to study the practice of teledermatology before, during, and after the COVID-19 pandemic. Methods: A literature search using the following keywords was done: online consultations, teledermatology, post-covid. Reports from integrated health care companies (eg, Practo) were also considered.

Results: According to the reports, Indians consulted physicians 10 times more during the second wave (April to May 2021) of the pandemic than in pre-COVID-19 times (January to February 2020). India experienced a record 30-fold spike in web-based physician consultations for COVID-19-related symptoms during this time, as compared to a 6-fold increase during the previous peak. More than $50 \%$ of all web-based consultations were for pulmonologists and general physicians for queries related to COVID-19 and the seasonal flu. Other key specialties that were consulted during this period included gynecology (10\%), dermatology $(8 \%)$, and pediatrics $(5 \%)$. The demand for general physicians and pulmonologists was at an all-time high, according to the data. Cutaneous manifestations were varied, and included urticaria, varicella-like vesicles, transient livedoid eruptions, livedoid vasculopathy, purpuric eruptions, lichenoid photodermatitis, erythroderma, photocontact dermatitis, and generalized pustular figurate erythema.

Conclusions: Continued advocacy efforts and future studies highlighting teledermatology's impact, particularly on minorities, underserved patient populations, and in resource-poor settings, are critical for long-term legislative changes to occur and to provide coverage to our most vulnerable patients. This presentation underscores the state of teledermatology prior to the pandemic, the legal statutory changes that permitted teledermatology to rapidly expand during the pandemic, and the significance of continued work after the pandemic. In short, the interruption of everyday life worldwide caused by SARS-CoV-2 has demonstrated that our method of practicing medicine needs reexamining.
\end{abstract}

Conflicts of Interest: None declared.

(iproc 2022;8(1):e36904) doi: 10.2196/36904

\section{KEYWORDS}

online consultations; telemedicine; teledermatology; COVID-19 
Edited by T Derrick; this is a non-peer-reviewed article. Submitted 28.01.22; accepted 28.01.22; published 18.02.22.

Please cite as:

Thomas $J$

The Practice of Teledermatology Before, During, and After the COVID-19 Pandemic iproc 2022;8(1):e36904

URL: https://www.iproc.org/2022/1/e36904

doi: $10.2196 / 36904$

PMID:

CJayarkar Thomas. Originally published in Iproceedings (https://www.iproc.org), 18.02.2022. This is an open-access article distributed under the terms of the Creative Commons Attribution License (https://creativecommons.org/licenses/by/4.0/), which permits unrestricted use, distribution, and reproduction in any medium, provided the original work, first published in Iproceedings, is properly cited. The complete bibliographic information, a link to the original publication on https://www.iproc.org/, as well as this copyright and license information must be included. 